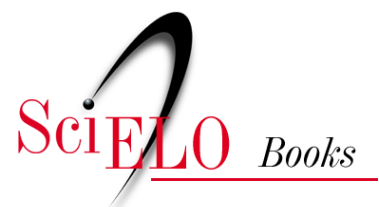

\title{
1 - 0 trabalho e a formação dos agentes comunitários de saúde e dos agentes indígenas de saúde
}

\author{
Luiza Garnelo \\ Sully de Souza Sampaio \\ Ana Lúcia Pontes
}

GARNELO, L., SAMPAIO, S.S., and PONTES, A.L. O trabalho e a formação dos agentes comunitários de saúde e dos agentes indígenas de saúde. In: Atenção diferenciada: a formação técnica de agentes indígenas de saúde do Alto Rio Negro [online]. Rio de Janeiro: Editora FIOCRUZ, 2019, pp. 13-24. Fazer saúde collection. ISBN: 978-65-5708-011-5. https://doi.org/10.7476/9786557080115.0002.

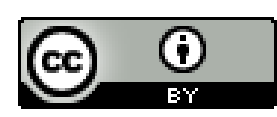

All the contents of this work, except where otherwise noted, is licensed under a Creative Commons Attribution $\underline{4.0 \text { International license. }}$

Todo o conteúdo deste trabalho, exceto quando houver ressalva, é publicado sob a licença Creative Commons Atribição 4.0.

Todo el contenido de esta obra, excepto donde se indique lo contrario, está bajo licencia de la licencia $\underline{\text { Creative }}$ Commons Reconocimento 4.0. 


\section{I}

\section{O trabalho e a formação dos agentes comunitários de saúde $e$ dos agentes indígenas de saúde}

As formações profissionais em saúde, ao tomarem o trabalho como eixo para a construção curricular, provocam reflexões acerca do papel social dos trabalhadores. Inicialmente, gostaríamos de destacar que o debate sobre o papel e a profissionalização dos AIS se articula com a trajetória dos trabalhadores de nível médio e fundamental da saúde. Esses trabalhadores estão inseridos na moderna divisão social do trabalho no mundo capitalista, a qual limitou sua atuação às atividades manuais e rebaixou seu trabalho ao plano da baixa complexidade. Fica subentendido que sua qualificação dispensa a inserção de fundamentos teóricos, as remunerações para tais cargos são baixas e eles ocupam uma posição subalterna na hierarquia dos profissionais da saúde.

Entretanto, principalmente a partir da década de 1980, o movimento de regulamentação das profissões de nível técnico e elementar potencializou a constituição do campo da educação profissional em saúde. As perspectivas críticas desse campo se contrapõem a essa visão naturalizada da divisão social do trabalho e partem de uma concepção na qual se compreende o trabalhador como sujeito transformador de sua realidade. É a partir dessa perspectiva que buscamos desenvolver a profissionalização dos AIS.

Porém, observamos que na atuação dos AIS, assim como Silva e Dalmaso (200 I : 180) descreveram no caso dos ACS, "o agente não dispõe de instrumentos, de tecnologia, de saberes para corresponder às expectativas das comunidades e dos governos". Sem uma definição da sua área de saber e de prática, os AIS encontram muitos desafios para seu reconhecimento profissional e para a qualidade e efetividade de suas ações nas comunidades onde atuam.

Para melhor compreensão das questões que perpassam esses desafios na formação profissional dos AIS, consideramos fundamental contextualizar como tem se construído 
o papel dos TCS no mundo, e, no Brasil, especificamente, dos ACS, pois seus marcos influenciaram diretamente a experiência em análise. Também faremos uma breve apresentação do subsistema de saúde indígena e do panorama da formação e trabalho dos AIS no Brasil.

\section{Trabalhadores comunitários da saúde: reflexões com base nas primeiras experiências implementadas no mundo}

Para revermos a atuação dos TCS durante o primeiro período de expansão da APS, usaremos dados da revisão internacional, publicada em 1990, intitulada Community Health Workers in National Programmes: just another pair of hands? (em português, Trabalhadores Comunitários em Saúde nos Programas Nacionais: apenas um par extra de mãos?). Essa retomada é importante para analisarmos as marcas históricas que tendem a persistir no papel desses trabalhadores e identificarmos os limites na atuação dos TCS sem profissionalização.

Os autores ressaltam que o termo trabalhadores comunitários de saúde foi difundido a partir de 1975 e agregou vários tipos e perfis de trabalhadores que tinham como característica comum serem pessoas provenientes e residentes das comunidades nas quais desenvolviam ações de saúde. A difusão da APS a partir da Conferência Internacional de Cuidados Primários à Saúde em 1978 levou vários países a promoverem programas de TCS, que tinham em comum dois objetivos: estender as ações de saúde para populações não assistidas e envolver membros das comunidades nesses programas (Walt et al., 1990).

Walt e colaboradores (1990) relatam que os primeiros programas de TCS foram inspirados em relatórios de profissionais da saúde que treinaram assistentes não profissionais para fazer tarefas vistas como de domínio profissional da saúde. Esses treinamentos eram curtos e focados na educação e prevenção, mas também incluíam a instrução sobre medicamentos, primeiros socorros, doenças específicas e tratamentos simples. Essas iniciativas eram tentativas de treinar um amplo número de trabalhadores da saúde, rapidamente e com baixo custo, para cuidar de comunidades dificilmente atendidas pelos serviços de saúde.

Os TCS eram treinados por curtos períodos durante os quais lhes era ensinado um número limitado de habilidades, mas no seu cotidiano eram solicitados a manejar os mais variados problemas e situações para as quais não estavam aptos, uma vez que 
frequentemente viviam em áreas remotas e o encaminhamento para outros serviços de saúde ou a supervisão por outros profissionais eram difíceis de garantir.

Walt e colaboradores (1990) analisaram alguns papéis comuns e contraditórios desempenhados pelos trabalhadores comunitários:

- Minidoctors: os TCS conseguiam maior credibilidade das comunidades quando eram capazes de tratar doenças e ofertarem medicamentos. Entretanto, os sistemas de saúde eram muito relutantes em permitir que os TCS tivessem acesso a essas técnicas, como uso de antibióticos e injeções.

- Um par extra de mãos: quando os TCS estavam ligados a um serviço de saúde, os profissionais de enfermagem tendiam a percebê-los como ajuda extra para dividir a carga de trabalho assistencial, executando tarefas que não desejavam realizar. Os TCS também tendiam a preferir trabalhar nos serviços de saúde ao lado de outro profissional do que realizar visitas domiciliares e atividades educativas.

- Educadores: a prioridade da atuação dos TCS deveria ser a atividade educativa, entretanto as evidências mostraram ser frequentes as ações voltadas para procedimentos. Os autores discutem que, além das dificuldades inerentes às tentativas de mudar o comportamento das pessoas e de atuar em problemas multifatoriais, é problemático manter o interesse da comunidade num conjunto limitado de atividades de educação, além da falta de incentivo e recursos para desenvolvê-las nos sistemas de saúde.

- Ligação: frequentemente esperava-se que os TCS respondessem ou consultassem comitês comunitários de saúde, organizações comunitárias e mantivessem laços próximos com cuidadores tradicionais e parteiras. Associa-se esse papel a uma expectativa de que eles efetuassem ligação entre os serviços, os profissionais e as comunidades, mas, por se identificarem mais com os profissionais da saúde, mesmo mantendo os vínculos comunitários, os TCS não exerceriam essa ligação com os terapeutas tradicionais.

Esses papéis eram influenciados pelos sistemas de saúde ao qual os TCS estavam vinculados, mas os autores observaram que os trabalhadores comunitários não eram muito ativos, a não ser que houvesse supervisão e estímulo constante. Em geral, os moradores das comunidades atendidas pelos TCS ficavam desapontados com o baixo número de atividade curativas que estes executavam, e os TCS, por sua vez, se sentiam inadequados e frustrados por não cumprirem essas expectativas. Naquele período havia poucos estudos sobre a qualidade e impacto em saúde do trabalho dos TCS. 
O outro desafio para o trabalho dos TCS se relacionava ao fato de que eles tinham o foco do seu trabalho no indivíduo e não na comunidade; eles aprendiam habilidades preventivas focadas em comportamentos individuais. Esse enfoque individual da doença é clássico no pensamento da saúde e ajuda a estruturar a formação e os serviços de saúde, mas limita a atenção dada a variáveis ambientais, sociais, culturais e econômicas que influenciam o comportamento das pessoas.

As experiências analisadas por Walt e colaboradores (1990) sugerem que os TCS não conseguiam suprir o vão entre comunidade e profissionais de APS, pois tendiam a se identificar com os profissionais e se tornavam o mais baixo nível da hierarquia profissional. Walt e colaboradores (1990) recomendam que para a qualidade de seu trabalho, os TCS precisam de treinamento sólido, educação continuada, suporte contínuo de medicamentos e apoio e supervisão constantes.

Veremos a seguir que, no contexto brasileiro, muitas dessas características e desafios se repetiram na trajetória dos ACS e dos AIS. Esse perfil de TCS foi também implementado no Brasil; por meio da ESF, a expansão da APS incorporou de forma consistente no SuS cerca de 260 mil ACS, que incialmente tinham como requisitos de trabalho saber ler e escrever, disponibilidade de tempo e espírito de solidariedade e liderança. Desde o fim dos anos 1990, ocorreu uma intensa mobilização política pela regulamentação profissional dos ACS, com o reconhecimento da profissão e aprovação de um referencial nacional de formação técnica para os ACS.

\section{Trabalhadores comunitários no Brasil e a regulamentação profissional dos ACS}

O marco para a expansão e incorporação no SUS dos TCS ocorre a partir de 1991, com o Programa de Agentes Comunitários de Saúde (Pacs), e, em I 994, com o Programa Saúde da Família (PSF), atual ESF. Nesse momento, o ACS passa ser considerado como estratégico para a mudança do modelo de atenção e se transforma no maior quantitativo de trabalhadores responsáveis pela expansão dos serviços, atualmente estimados em mais de 260 mil.

O processo de trabalho e de formação dos ACS é perpassado por diversas disputas. Uma das questões colocadas se refere ao contexto de redução do papel do Estado, que tenciona a limitação da APS a um conjunto de ações e serviços, em contraposição à compreensão da APS como porta de entrada do sistema, com garantia de acesso integral à saúde (Morosini, Corbo E Guimarães, 2007). Outra questão importante se relaciona ao contexto de precarização dos vínculos de trabalho. 
Desde o começo da implantação do Pacs e do PSF, consolidou-se a ideia de que o ACS seria um mediador entre a comunidade e os serviços de saúde, principalmente com o objetivo de levar informações biomédicas para a população e subsidiar os demais profissionais com dados do contexto sociocultural e familiar. Nessa construção do papel social do ACS, perpassa a noção de que, por pertencer ao mesmo contexto sociocultural, teria a capacidade natural de interagir em estilo e linguagem mais efetivos, garantindo melhores resultados das ações de saúde, perspectiva da qual somos críticos. A defesa e normalização dessa perspectiva serviram de justificativa para que, de |99| até 2002, os critérios para seleção de ACS se limitassem a: ser maior de 18 anos, saber ler e escrever, ter disponibilidade de tempo e capacidade de liderança e solidariedade. Segundo Morosini, Corbo e Guimarães (2007: 267),

A (não) escolarização dos ACS, justificada pela necessidade de esse trabalhador ser representativo da comunidade em que atua, denota a desvalorização da dimensão conceitual de sua qualificação, o que fortalece a desvalorização social desse trabalhador, sustenta a sua baixa remuneração e contrapõe-se à pauta política por uma melhor qualificação dos trabalhadores de saúde, de uma maneira geral.

Outro marcador da desvalorização desses trabalhadores é dado pelos seus recorrentes relatos de desvio de função, denotando a ideia de que o trabalho do ACS é "inespecífico e de baixa complexidade, podendo ser redirecionado, segundo as necessidades dos serviços, para atividades que supostamente exigem pouca capacitação" (Morosini, Corbo \& Guimarães, 2007: 267).

A primeira diretriz para trabalho dos ACS foi a portaria n. 1.886, em 1997, que caracteriza sua atuação como centrada na promoção da saúde e na prevenção de doenças; ademais vedava ao ACS o trabalho interno nas unidades de saúde. Segundo Morosini, Corbo e Guimarães (2007), entre 1998 e 1999 foram apresentados três projetos de lei para regulamentar o trabalho do ACS, e o decreto n. 3.189, de outubro de 1999, fixou as diretrizes para exercício da atividade dos ACS: "cabe ao Agente Comunitário de Saúde (ACS) (...) desenvolver atividades de prevenção de doenças e promoção da saúde, por meio de ações educativas individuais e coletivas, nos domicílios e na comunidade, sob supervisão competente".

Em razão da consistente atuação do movimento dos ACS, em 2002 aprovou-se a lei n. 10.507, que os reconhece como profissionais do SUS para o "desenvolvimento ações de promoção da saúde e prevenção das doenças por meio de processos educativos em saúde, privilegiando o acesso às ações e serviços de informação e promoção social e de proteção e desenvolvimento da cidadania, no âmbito social e da saúde" (Brasil, 2004: 17). Também se determinou com essa lei que os ACS a partir daquele momento 
deveriam ter o ensino fundamental completo. A continuidade e profundidade do debate desencadeado nesse período estabeleceram a convicção de que esse trabalhador desenvolvia um trabalho complexo e que sua formação não deveria se limitar a uma qualificação inicial (Morosini, Corbo \& Guimarães, 2007). Assim, em 2004, foi discutido e aprovado o Referencial Curricular para Curso Técnico de Agente Comunitário de Saúde (Brasil, 2004), que propôs um itinerário formativo que articulasse elevação da escolaridade e profissionalização de nível técnico em saúde e estabelecesse seis competências para a formação dos ACS:

I) Desenvolver ações que busquem a integração entre as equipes de saúde e a população adstrita à unidade básica de saúde, considerando as características e as finalidades do trabalho de acompanhamento de indivíduos e grupos sociais ou coletividades.

2) Realizar, em conjunto com a equipe, atividades de planejamento e avaliação das ações de saúde no âmbito de adstrição da unidade básica de saúde.

3) Desenvolver ações de promoção social e de proteção e desenvolvimento da cidadania no âmbito social e da saúde.

4) Desenvolver, em equipe, ações de promoção da saúde visando à melhoria da qualidade de vida da população, à gestão social das políticas públicas de saúde e ao exercício do controle da sociedade sobre o setor da saúde.

5) Desenvolver ações de prevenção e monitoramento dirigidas a grupos específicos e a doenças prevalentes, conforme definido no plano de ação da equipe de saúde e nos protocolos de saúde pública.

6) Desenvolver ações de prevenção e monitoramento dirigidas às situações de risco ambiental e sanitário para a população, conforme plano de ação da equipe de saúde (Brasil, 2004).

Em 2006, no intuito de avançar a desprecarização do trabalho dos ACS, os requisitos para exercício da atividade de ACS e seu processo de contratação foram revistos na lei n. I I.350. Em termos de atribuições do trabalho das equipes de Saúde da Família, o documento mais recente se encontra na portaria n. 2.436, de 21 de setembro de 2017, que aprova a Política Nacional de Atenção Básica (Pnab), estabelece a revisão de diretrizes e normas para a organização da atenção básica, para a ESF e o Pacs. Como atribuições específicas dos ACS:

I - Trabalhar com adscrição de indivíduos e famílias em base geográfica definida e cadastrar todas as pessoas de sua área, mantendo os dados atualizados no sistema de informação 
da atenção básica vigente, utilizando-os de forma sistemática, com apoio da equipe, para a análise da situação de saúde, considerando as características sociais, econômicas, culturais, demográficas e epidemiológicas do território, e priorizando as situações a serem acompanhadas no planejamento local;

II - Utilizar instrumentos para a coleta de informações que apoiem no diagnóstico demográfico e sociocultural da comunidade;

III - Registrar, para fins de planejamento e acompanhamento das ações de saúde, os dados de nascimentos, óbitos, doenças e outros agravos à saúde, garantido o sigilo ético;

IV - Desenvolver ações que busquem a integração entre a equipe de saúde e a população adscrita à UBS, considerando as características e as finalidades do trabalho de acompanhamento de indivíduos e grupos sociais ou coletividades;

V - Informar os usuários sobre as datas e horários de consultas e exames agendados;

$\mathrm{VI}$ - Participar dos processos de regulação a partir da atenção básica para acompanhamento das necessidades dos usuários no que diz respeito a agendamentos ou desistências de consultas e exames solicitados;

VII - Exercer outras atribuições que Ihes sejam atribuídas por legislação específica da categoria, ou outra normativa instituída pelo gestor federal, municipal ou do Distrito Federal. (Brasil, 2017)

Se no cenário urbano do trabalho e formação do ACS existem esses diversos desafios, no contexto indígena veremos que se agregam outras questões e complexidades, que iremos abordar a seguir.

\section{A estruturação da APS para os povos indígenas e os AIS}

No Brasil realizou-se, em 1986, a I a Conferência para Proteção à Saúde dos Povos Indígenas, que iniciou a primeira formulação de uma política pública nacional de atenção à saúde dos povos indígenas. Historicamente, apenas ações pontuais e emergenciais de saúde eram ofertadas aos povos indígenas, o que entre outros fatores (como o avanço em seus territórios) gerou um quadro sanitário alarmante nessa população.

Durante a década de 1980, em razão da dificuldade de acesso aos serviços de saúde e baixa permanência das equipes de saúde da Fundação Nacional de Saúde (Funasa) e do Ministério da Saúde (MS) nas aldeias, universidades e organizações não governamentais realizaram projetos de formação e capacitação de índios para desenvolverem ações de saúde nas comunidades. Essas experiências criaram a figura do AIS, que seria um membro da comunidade, indicado por ela para desenvolver ações de saúde. 
A Constituição de 1988 foi um marco na mudança da relação do Estado com os povos indígenas brasileiros, pois assegura aos índios sua organização social, costumes, línguas, crenças e tradições, e reconhece a demarcação e usufruto exclusivo de seus territórios. No âmbito das políticas sociais, as lutas dos povos indígenas no Brasil possibilitaram a implantação de um sistema público específico de atenção à saúde dos povos indígenas integrado ao SUS. A criação de um subsistema - único dessa natureza entre os produtos advindos da Reforma Sanitária - expressa o reconhecimento, pelo legislador, das limitações de uma política universalizante como a do SUS, diante das especificidades e necessidades das minorias indígenas. Buscava-se, assim, garantir tanto o direito ao acesso universal aos serviços de saúde, equalizando o índio aos outros cidadãos brasileiros, quanto o cuidado culturalmente específico e sensível às necessidades locais das múltiplas etnias atendidas pelo subsistema (Subsistema de Atenção à Saúde Indígena - Sasi/SUS).

Entretanto, existem diversos limites e dificuldades para a plena implementação de uma atenção à saúde de qualidade e que respeite as especificidades socioculturais dos povos indígenas, tais como a insatisfação dos indígenas, a baixa resolutividade, e as inadequações culturais do subsistema.

A Política Nacional de Atenção à Saúde dos Povos Indígenas (Pnaspi) (Brasil, 2002) procurou assegurar o acesso à atenção integral à saúde, organizada sob a forma de DSEI, sob responsabilidade de um gestor federal, que inicialmente foi a Funasa/MS e, desde 2010 , é a Secretaria Especial de Saúde Indígena (Sesai/MS).

O modelo de atenção proposto se baseia numa rede interiorizada de atenção básica articulada com o restante da rede de serviços de saúde do SUS, para garantir assistência de média e alta complexidade. A estrutura do DSEI compreende os postos de saúde nas aldeias, onde atuam os AIS; os polos-base, primeiro nível de referência para os postos de saúde, com equipes multidisciplinares compostas de médicos, enfermeiros e técnicos de enfermagem; e a Casa de Saúde do Índio (Casai), que apoia as atividades de referência para o atendimento de média e alta complexidade nos demais serviços da rede do SUS. Dessa forma, a organização do distrito prevê diferentes níveis de resolutividade e se articula com o restante da rede SUS quando se faz necessária a realização de exames, de consultas com especialistas, de internações ou de procedimentos invasivos. Com a implementação dos DSEls, desde 1999, os AIS foram incorporados formalmente nas equipes multiprofissionais de saúde indígena (Emsi).

A Pnaspi frisa a importância da sensibilidade cultural no desenvolvimento das ações de saúde nos DSEls, tendo optado pelo termo atenção diferenciada para expressá-la. 
Em que pese a importância da atenção diferenciada na política de saúde dirigida às minorias indígenas, não há, no texto da Pnaspi, uma caracterização precisa de seu sentido nem indicação de formas concretas que essa diferenciação poderia assumir nas práticas sanitárias cotidianamente desenvolvidas nos DSEls.

A temática da atenção diferenciada e da articulação entre saberes científicos e tradicionais indígenas guarda interfaces com atributos da APS, na medida em que implica o reconhecimento e a construção das ações locais em articulação com as singularidades étnico-culturais e a diversidade nos modos de interpretar a doença, produzir estratégias de cura e cuidados e de mobilizar redes sociais e familiares para lidar com tais eventos. Nesse contexto, o AIS, na condição de membro indígena da equipe de saúde, desponta como elemento-chave, e essa é uma das dimensões principais com a qual pretendemos contribuir, demonstrando algumas estratégias e experiências desenvolvidas no curso técnico para AIS que podem ser adaptadas e utilizadas em outros contextos.

\section{Ambiguidades e indefinições na construção do papel do AIS}

Existem poucos dados sobre as características dos trabalhadores AIS no Brasil. Sabemos que seu quantitativo vem aumentando: em 1998, eram I.400; e em 2004 já eram 3.665 (Brasil, 2005). Com base em informações provindas de reuniões com a Sesai, estimamos que atualmente sejam ao redor de seis mil AIS e Agentes Indígenas de Saneamento (Aisan) contratados nos 34 DSEls existentes no Brasil.

Os AIS são os principais responsáveis pela regularidade do acesso a ações e serviços de saúde para cerca de 700 mil pessoas atendidas pelo subsistema de saúde indígena brasileiro. Esse subsistema foi criado em 1999, por meio da Lei Arouca (lei n. 9.836/99) que instituiu o Sasi e definiu que a organização da atenção à saúde se daria por redes territorializadas de saúde, na forma de 34 DSEls.

Ao revisarmos a trajetória e pesquisas sobre os AIS no Brasil, observamos vários pontos de convergência com a análise de Walt e colaboradores (1990). Nas revisões (Diehl, Langdon \& Dias-Scopel, 2012), aponta-se que, em geral, os AIS não possuem formação prévia e nenhuma exigência de escolaridade para iniciar seu trabalho (esse último aspecto tem mudado mais recentemente). Na literatura, os processos formativos nos distritos foram descritos como irregulares, intermitentes, sem certificação ou articulação com o processo de escolarização. A falta de formação e qualificação aparece como um agravante na relação com a equipe multidisciplinar, que o entende como um profissional não técnico e desqualificado. Assim, os AIS demandam e buscam individualmente acesso a conhecimentos em saúde. 
Os AIS têm diferentes perfis de atuação em cada região e DSEI, mas identificamos uma forte tendência na aplicação e distribuição de tecnologias biomédicas. Diehl, Langdon e Dias-Scopel (2012) avaliam que, nos contextos indígenas mais isolados, os AIS desempenham um papel mais claro na assistência e que, nos distritos com contato regular com os demais profissionais da equipe multiprofissional, as atribuições dos AIS são ainda menos definidas.

Encontramos no trabalho do AIS um conflito de expectativas e interesses. Para os enfermeiros e técnicos de enfermagem, os agentes não deveriam executar as ações técnicas, mas tão somente o preenchimento de fichas, distribuição de medicamento e atividades de comunicação. Entretanto, a expectativa das comunidades seria de que os agentes executassem ações biomédicas. Discute-se o papel de articulação/vinculação/ mediação entre os serviços de saúde e as práticas tradicionais como uma atribuição do AIS, mas aponta-se que essa função não é plenamente desenvolvida.

Todas essas pesquisas indicam que a implantação de um subsistema de saúde específico para os povos indígenas, com a inclusão de membros indígenas nas equipes, os próprios AIS, não garantiu o acesso a uma atenção à saúde de qualidade nem o protagonismo indígena desejado no campo da saúde indígena. Adiciona-se a isso o fato de que algumas lideranças indígenas apontam claramente que a implementação do subsistema de saúde indígena, ao invés de fortalecer o papel dos AIS (que já atuavam como voluntários há vários anos), fez com que eles perdessem autonomia e recursos para resolução dos problemas das comunidades.

Não obstante, os AIS representam uma melhoria de acesso aos serviços de saúde para os indígenas acompanhados. No modelo proposto no subsistema de saúde indígena, o AIS é central para a efetivação, no cotidiano, da atenção. Em razão da alta rotatividade dos profissionais não indígenas (Diehl, Langdon $\varepsilon$ Dias-Scopel, 20I2), em muitos distritos somente o AIS está atuando regularmente nas comunidades, principalmente nas mais isoladas. Portanto, em termos de ampliação de acesso, eles são fundamentais. Ademais, a perspectiva de construção da atenção diferenciada, dimensão relativa à especificidade e à diversidade sociocultural dos povos indígenas, está fortemente baseada na ação do AIS (Diehl, Langdon E Dias-Scopel, 2012).

Em 2012, a Sesai realizou um levantamento com as coordenações locais dos 34 DSEls para realizar um diagnóstico do perfil dos AIS no Brasil, cujos resultados foram divulgados em reunião. A secretaria obteve resposta de 31 distritos, e como perfil dos AIS naquele momento observaram-se: 
- 33\% dos AIS são do sexo feminino e 67\% do sexo masculino;

- Em relação à faixa etária: II\% com idade entre 18 e 25 anos, 19\% entre 25 e 30 anos, 22\% entre 30 e 35 anos, 19\% entre 35 e 40 anos e 28\% maiores de 40 anos;

- Em relação ao idioma, 7\% não falavam português;

- $88 \%$ têm alguma escolaridade e, desses, $46 \%$ têm ensino fundamental completo e $30 \%$ ensino médio, e $1 \%$ ensino superior;

- 55\% mencionaram realizar um curso introdutório dos AIS;

O dado que mais se destaca é a aparente tendência de elevação da escolaridade dos agentes, o que indica um contexto positivo para a profissionalização em nível técnico desses trabalhadores.

Em termos legais, até 2010, a Funasa/MS era a responsável pelo Sasi. Em dezembro de 2010 , foi criada a Sesai no MS, que assumiu essa responsabilidade, mas que, somente em 2017, apresentou novas diretrizes para a formação de AIS e Aisan.

Dessa forma, os documentos nos quais pudemos nos basear para discussão da regulamentação profissional dos AIS ainda eram do período da Funasa. A Funasa (Brasil, 2005) apontava como papel do AIS:

- acompanhamento de crescimento e desenvolvimento;

- acompanhamento de gestantes;

- atendimento aos casos de doenças mais frequentes (infecção respiratória, diarreia, malária);

- acompanhamento de pacientes crônicos;

- primeiros socorros;

- promoção da saúde e prevenção de doenças de maior prevalência;

- acompanhamento da vacinação; e

- acompanhamento e supervisão de tratamentos de longa duração.

Essas atribuições são genéricas e inespecíficas e não demonstram consideração ao contexto indígena. Em razão da falta de clareza quanto ao papel e atribuições do AIS, os diversos DSEls por vezes apresentam alguma regulamentação própria. Assim, encontramos nas diversas regiões diferentes perfis profissionais dos AIS.

$\mathrm{Na}$ falta de uma regulamentação específica, considerando o contexto histórico e inserção na atenção básica dos AIS, optamos pela utilização de documentos e 
regulamentações propostas para os ACS, em razão das semelhanças históricas e de construção do papel social desses trabalhadores.

Durante a implementação da proposta formativa, houve reuniões regulares da equipe de coordenação do curso com as lideranças indígenas e com a equipe de profissionais e gestores do DSEl do Alto Rio Negro. Com base nessas conversas, percebemos a necessidade de que o desenvolvimento curricular permitisse a melhor definição do papel dos AIS nas equipes e comunidades, com detalhamento das técnicas e atividades a serem desenvolvidas em cada área de atuação. Assim, durante a implementação curricular do CTACIS, podemos identificar um conjunto de atribuições que, de acordo com a coordenação do curso, os técnicos em agente comunitário indígena de saúde poderiam desenvolver, em conjunto e sob supervisão da equipe multidisciplinar de saúde indígena, conforme o perfil e necessidade de cada comunidade. 